\title{
Study of Osteomyelitis in paediatric patients
}

\author{
Sindhur P.K' ${ }^{1}$, Razak S. A ${ }^{2}$, Sandesh K.S ${ }^{3}$, Shah $\mathbf{P}^{4}$, Singhal $\mathbf{A}^{5}$ \\ ${ }^{1}$ Dr. Praveenkumar Sindhur, Consultant Pediatrician, Department of Pediatrics, ${ }^{2}$ Dr. Shadiya A Razak, Postgraduate \\ Student, Department of Pediatrics, ${ }^{3}$ Dr. Sandesh KS, Department of PG Studies in Social Work, Nehru Memorial \\ College, KVG Medical College, Sullia, DK, Karnataka, ${ }^{4}$ Dr. Pooja Shah, Lecturer, Department of Microbiology, \\ SBHGMC Dhule, Maharashtra, ${ }^{5}$ Dr. Anish Singhal, Assistant Professor, Department of Physiology, KVG Medical \\ College, Sullia, DK, Karnataka, India.
}

Address for Correspondence: Dr. Anish Singhal, Email: drsandesh2@gmail.com

\begin{abstract}
Introduction: Osteomyelitis is defined as inflammation of bone. Though the main culprits are bacteria occasionally fungi may also be involved. Prognosis of untreated or delayed treatment onset osteomyelitis is often bad and leads to adverse outcomes. Very few cases of osteomyelitis are reported from India as compared to other infectious conditions. In this study we aim to study the number of patients, male: female ratio, type of osteomyelitis and the bacterial profile associated with osteomyelitis. Methods: After obtaining Institutional Ethics approval, the present study was conducted from $1^{\text {st }}$ January $2016-31^{\text {st }}$ December 2016 at KVG Medical College, Sullia. Blood and sample like fluid aspirate or discharge collected was subjected to microscopy and culture. Antibiotic susceptibility testing was done using disk diffusion methods. Radiological data of the patients was also collected. Results: Bacteria were isolated from 32 cases. The most affected age group was of 6-10 years of age (46.87\%). Males were more affected as compared to females and male: female ratio was 3:1. Maximum number of patients were of acute osteomyelitis (87.5\%) followed by subacute osteomyelitis (9.38\%) and chronic osteomyelitis was found in just a single patient (3.12\%). Long bones like tibia $(56.25 \%)$, femur $(18.75 \%)$, humerus $(12.5 \%)$ were mostly affected. Blood culture was positive in $65.62 \%$ cases. Methicillin Sensitive Staphylococcus Aureus (MSSA) was the most common isolate (56.25\%) followed by, Methicillin Resistant Staphylococcus Aureus (MRSA), Acinetobacter, Coagulase Negative Staphylococcus (CONS), Streptococcus pyogens, E.coli and Pseudomonas. Conclusion: Thus, S. aureus was the most common cause of acute bacterial osteomyelitis and mostly affected school age group children.
\end{abstract}

Key words: Osteomyelitis, Staphylococcus aureus, Organism

\section{Introduction}

The term osteomyelitis refers to a broad group of diseases characterized by inflammation or infection of the bone and/or bone marrow [1]. Osteomyelitis was first described by Chassaignac in the year 1852. The word "osteomyelitis" is derived from the words osteon (meaning bone) and muelinos (meaning marrow) [2]

The pathophysiology behind this is the inoculation of metaphyseal vessels at the transition point of arteriolar vessels to the venous sinusoids slowing down the blood flow and increasing the vascular turbulence [3]. Osteomyelitis is mainly divided into 3 types: Acute, subacute and chronic type. Acute osteomyelitis presents

Manuscript received: $24^{\text {th }}$ February 2017

Reviewed: $2^{\text {nd }}$ March 2017

Author Corrected: $9^{\text {th }}$ March 2017

Accepted for Publication: $15^{\text {th }}$ March 2017 with $<2$ weeks' duration, subacute osteomyelitis from 2 weeks- 3 months and chronic osteomyelitis is for $>3$ months [4].

The causes of osteomyelitis may be secondary to a contiguous focus of infection after trauma, surgery, or insertion of a prosthetic joint; or secondary to a contiguous focus of infection associated with vascular insufficiency, primarily occurring in patients with diabetes mellitus and/or peripheral vascular disease and following hematogenous spread of infection, which is major mechanism for vertebral osteomyelitis and in children [5]. According to literature, S. aureus, Enterobacter spp., Streptococcus (group A and B), Haemophilus influenzae are most commonly involved causative agents in pediatric osteomyelitis [6]. Bacteria 
like Mycobacterium, Bartonella, Coxiella, fungi (Histoplasma,Cryptococcus and Blastomyces, Candida) are amongst rare causes of osteomyelitis and are seen in patients who are immunocompromised or who have the history of travel to an endemic area [7].

The common clinical features are fever, pain at the site of infection, reluctance and difficulty to use affected extremity. Less common clinical manifestations are anorexia, malaise and vomiting. Focal swelling, tenderness, warmth, and erythema are usually present on examination [8].

Drainage of purulent material may take place with fistula. Early diagnosis and prompt treatment leads to very less mortality (around zero) and the complication rate around $5 \%[9]$.

\section{Aim}

1) To study the number of patients of osteomyelitis in the given setting.

2) To study male: female ratio.

3 ) To study type of osteomyelitis.

4) To study the bacterial profile associated with osteomyelitis.

\section{Material and Methods}

The present study was conducted from $1^{\text {st }}$ January 2016 - $31^{\text {st }}$ December 2016 at KVG Medical College, Sullia. Institutional Ethics Committee approval was obtained. Pediatric patients coming with the complaints of fever, bone pain with clinical diagnosis of osteomyelitis were included in the study. Study was performed only with informed consent from the patients/legal guardian.

Patients were examined for signs like tenderness, oedema or warmth. $\mathrm{X}$ ray or computerized tomography (CT) scan of the patients was done and data was collected. Specimens like, discharge from that site or fluid by aspiration were collected depending on the case presentation.

Blood culture was done in all the patients. Samples were sent for Gram staining and culture. Culture was done on blood agar and MacConkey agar.

Conventional methods (biochemical tests) were used for bacterial identification. Antibiotic Susceptibility testing was performed using disk diffusion method. Treatment was decided and started based on the reports of Antibiotic susceptibility tests.

\section{Results}

In our 1 year duration, there were 32 cases presenting with signs and symptoms of osteomyelitis i.e. clinical osteomyelitis. We could isolate bacteria in all the cases.

Table-1: Age Distribution in cases of Osteomyelitis.

\begin{tabular}{|c|c|c|}
\hline Age (Years) & Number (n=32) & Percentage (\%) \\
\hline $\mathbf{0 - 5}$ & 9 & 28.13 \\
\hline $\mathbf{6 - 1 0}$ & 15 & 46.87 \\
\hline $\mathbf{1 0 - 1 5}$ & 8 & 25 \\
\hline Total & 32 & $100 \%$ \\
\hline
\end{tabular}

Maximum number of cases (46.87\%) were seen in 6-10 years' age group.

Table-2: Sex distribution in patients presenting with Osteomyelitis.

\begin{tabular}{|c|c|c|c|}
\hline Sex & Number $(\mathbf{n}=\mathbf{3 2})$ & Total Admissions & Incidence \\
\hline Males & $24(75 \%)$ & $2077(52.72 \%)$ & $1.15 \%$ \\
\hline Females & $8(25 \%)$ & $1862(47.27)$ & $0.42 \%$ \\
\hline Total & $32(100 \%)$ & $3939(100 \%)$ & $0.812 \%$ \\
\hline
\end{tabular}

Males were affected 3 times more as compared to females. Overall incidence was found to be $0.812 \%$. 
Table-3: Type of Osteomyelitis.

\begin{tabular}{|c|c|c|}
\hline Type of osteomyelitis & Number(n=32) & Percentage (\%) \\
\hline $\begin{array}{c}\text { Acute } \\
\text { (<2 weeks duration) }\end{array}$ & 28 & 87.5 \\
\hline $\begin{array}{c}\text { Sub-acute } \\
\text { (2weeks-3 months) }\end{array}$ & 3 & 9.38 \\
\hline $\begin{array}{c}\text { Chronic } \\
\text { ( 3 months) }\end{array}$ & 1 & 3.12 \\
\hline Total & 32 & $100 \%$ \\
\hline
\end{tabular}

Acute osteomyelitis was the most common type in our study $(87.5 \%)$.

\section{Site of infection}

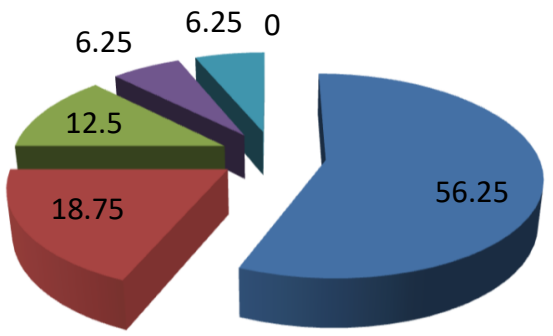

- Tibia

- Femur

Humerus

Fibula

Foot

Multiple sites

Figure-1: Site of Infection

Tibia was the most commonly affected (56.25\%) site followed by femur (18.75\%).

Table-4: Samples on Blood Culture.

\begin{tabular}{|c|c|c|}
\hline Blood culture result & Number(n=32) & Percentage \\
\hline Positive & 21 & 65.62 \\
\hline Negative & 11 & 34.38 \\
\hline Total & 32 & $100 \%$ \\
\hline
\end{tabular}

Thus, $65.62 \%$ cases were positive by blood culture while $34.38 \%$ cases were negative.

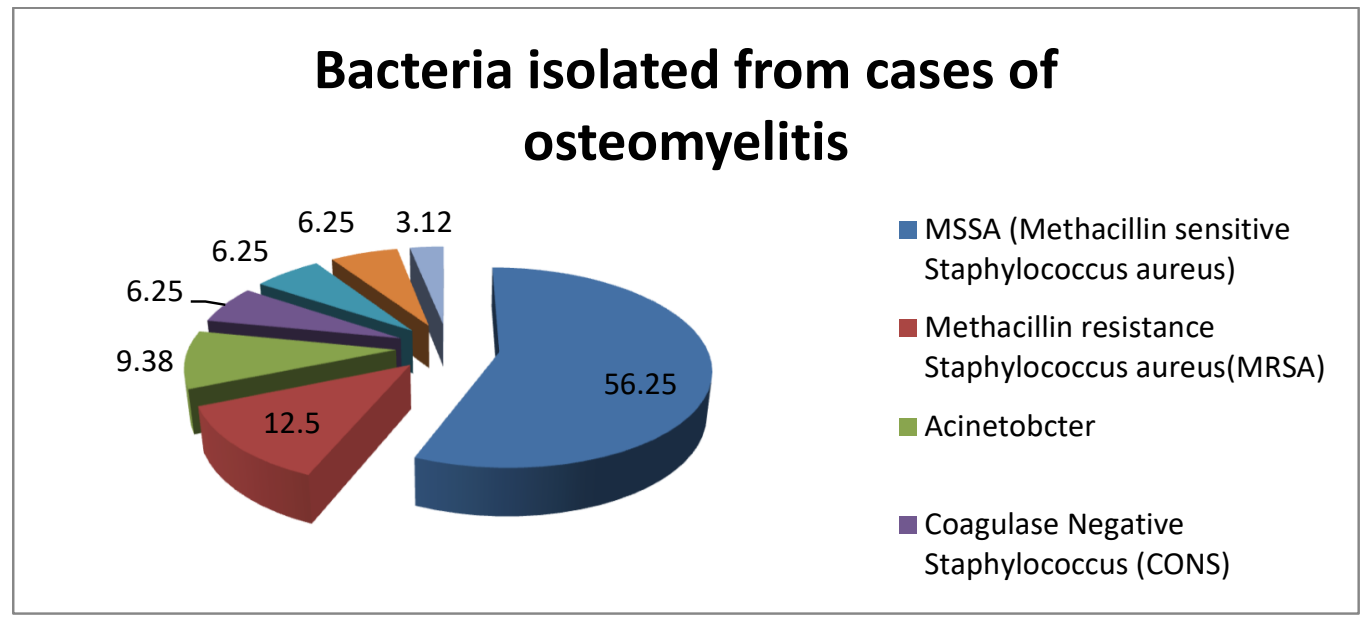

Figure-2: Bacteria isolated from cases of Osteomyelitis 
$M S S A$ was the most commonly isolated, in about $56.25 \%$ cases. MRSA was isolated in $12.5 \%$ cases, Acinetobacter in 9.38\%, CONS, S.pyogens and E.coli were isolated in 6,25\% each. Pseudomonas was isolated in $3.12 \%$.

\section{Discussion}

In our study 6-10 years age group was the most affected $(46.87 \%)$, as seen in table 1 . This finding correlates well with the study of Puccini et al who found maximum number of cases in 6-14 years' age group $(61.9 \%)$ cases [10]. This finding also correlate with Zaoutis et al, who studied children aged 2 months to 17 years and found detection rate of $42 \%$ in $<5$ years and $58 \%$ for $6-17$ years. As per results seen in table 2, males were more affected (75\%) as compared to females $(25 \%)$. Similar findings were seen in other studies [10] and reviews [11]. Our study reports an overall incidence of $0.812 \%$ pediatric osteomyelitis.

Cases of acute osteomyelitis (Table 3) were much more $(87.5 \%)$ as compared to subacute $(9.38 \%)$ and chronic osteomyelitis $(3.12 \%)$. This may be due to the fact that in our study, $28.13 \%$ cases were of $<5$ years and in this age group it is difficult to elicit pain as compared to older age group.

This brings the older pediatric age group ( $>5$ years) more quickly to hospital as compared to younger group. A western study mentioned that acute osteomyelitis is seen more commonly among children [12].

We found (Figure 1) tibia (56.25\%), femur (18.75\%) and humerus $(12.5 \%)$ were more involved as compared to other bones. The reason for such a finding could be due the possibility of these bones being more prone to injury as compared to other bones. This finding correlates well with a similar study by Puccini et al [10]. Two cases of foot osteomyelitis found in our study had a history of trauma.

Blood culture was found to be positive in $65.52 \%$ cases (Table-4), which as per literature is able to detect around $50 \%$ cases [13]. Discharge from the site have more detection rate [10].

Like other studies also, S.aureus was most common isolate $(68.75 \%)$ amongst cases (Figure 2). The reason is, S.aureus adheres to bone by expressing receptors (adhesins) for components of bone matrix (fibronectin, laminin, collagen and bone sialo glycoprotein); the expression of the collagen-binding adhesion permits the attachment of the pathogen to cartilage [5] Acinetobacter, E. coli and Pseudomonas were the Gram-Negative bacteria that were isolated.

\section{Conclusion}

S.aureus was the most common cause of acute bacterial osteomyelitis and mostly affected school age group children.

\section{List of Abbreviations}

MSSA: Methicillin Sensitive Staphylococcus Aureus

MRSA: Methicillin Resistant Staphylococcus Aureus

CONS: Coagulase Negative Staphylococcus

S.pyogens: Streptococcus Pyogens

E. coli: Escherichia Coli

Funding: Nil, Conflict of interest: None initiated, Perission from IRB: Yes

\section{References}

1. Mayank Roy, Jeremy S. Somerson, Kevin G. Kerr and Jonathan L. Conroy. Osteomyelitis. Mauricio S. Baptista and Joao Paulo Tadivo. Open access; InTech; 2002. ISBN: 978-953-51-0399-8.

2. Lew DP, Waldvogel FA. Osteomyelitis. N Engl J Med. 1997 Apr 3;336(14):999-1007.

3. Jansson A, Jansson V, von Liebe A. [Pediatric osteomyelitis]. Orthopade. 2009 Mar;38(3):283-94. doi: 10.1007/s00132-008-1402-6.

4. Ronald M. Laner and Carol B. Lindsley. Infectious arthritis and osteomyelitis. Section 5, Chapter 37Arthritis related to infection. Available from: http:// peds.stanford.edu/Rotations/red_team/pdfs/JIA/Cassidy - Petty \%20 Infectious \%20 Arthritis \%20 and \%20 Osteomyelitis.pdf.

5. Diana Gomes, Margarida Pereira, Ana Francisca Bettencourt. Osteomyelitis: an overview of antimicrobial therapy. Brazilian Journal of Pharmaceutical Sciences. 2013; 49 (1): 13-27. Doi: 10. 1590 / S1984-825020 13000100003

6. Lew DP, Waldvogel FA. Osteomyelitis. Lancet. 2004 Jul 24-30;364(9431):369-79.

7. Gutierrez K. Bone and joint infections in children. Pediatr Clin North Am. 2005 Jun;52(3):779-94, vi. 
8. Dich VQ, Nelson JD, Haltalin KC. Osteomyelitis in infants and children. A review of 163 cases. Am J Dis Child. 1975 Nov;129(11):1273-8.

9. Darville T, Jacobs RF. Management of acute hematogenous osteomyelitis in children. Pediatr Infect Dis J. 2004 Mar;23(3):255-7.

10. Pedro Fiorini Puccini, Maria Aparecida G. Ferrarini, Antônio Vladir Iazzetti. Pediatric acute hematogenous osteomyelitis: analysis of patients assisted in a university hospital. Rev Paul Pediatr. 2012;30(3):353-8. DOI: $10.1590 / \mathrm{S} 0103-05822012000300008$.
11. Peltola H, Pääkkönen M. Acute osteomyelitis in children. N Engl J Med. 2014 Jan 23; 370(4):352-60. doi: 10.1056/NEJMra1213956.

12. Harik NS, Smeltzer MS. Management of acute hematogenous osteomyelitis in children. Expert Rev Anti Infect Ther. 2010 Feb;8(2):175-81. doi: 10.1586/ eri.09.130

13. Hatzenbuehler J, Pulling TJ. Diagnosis and management of osteomyelitis. Am Fam Physician. 2011 Nov 1; 84(9):1027-33.

\section{How to cite this article?}

Sindhur P.K, Razak S.A, Sandesh K.S, Shah P, Singhal A. Study of Osteomyelitis in paediatric patients. J PediatrRes.2017;4(03):219-223.doi:10.17511/ijpr.2017.i03.04. 